\title{
Impaired skeletal formation in mice overexpressing DMPI
}

\author{
This article was published in the following Dove Press journal: \\ Orthopedic Research and Reviews \\ 16 September 2009 \\ Number of times this article has been viewed
}

\section{Michael Albazzaz \\ Karthikeyan Narayanan \\ Jianjun Hao \\ Roma Andheri \\ Amsaveni Ramachandran \\ Sriram Ravindran \\ Anne George}

Brodie Tooth Development Genetics and Regenerative Medicine Research Laboratory, University of Illinois, Chicago, IL, USA
Correspondence: Anne George Brodie Tooth Development Genetics and Regenerative Medicine Research Laboratory, University of Illinois at Chicago, Chicago, IL, 606 I2, USA Tel + I 312492882 I

Fax + I 3I29966044

Email anneg@uic.edu

\begin{abstract}
Dentin matrix protein 1 (DMP1) is a noncollagenous protein expressed in mineralized tissues such as bone, dentin, and cartilage. To investigate the role of DMP1 during bone formation, transgenic mice overexpressing DMP1 under the control of the CMV promoter were generated. These mice displayed an increased mineralization phenotype in bone. In addition, accelerated terminal differentiation of the epiphyseal growth plate chondrocytes were also observed. To investigate the potential role of DMP1 in osteoblast differentiation, bone marrow stem cells were stimulated with DMP1 and assayed for "early" and "late" markers for osteoblast differentiation. DMP1 treatment increased the expression of CBFA1, BMP2, COL1, and OCN within two days. An in vitro mineralized nodule formation assay demonstrated that the bone marrow stem cells could differentiate and form a mineralized matrix in the presence of DMP1. Together, these results support a model whereby DMP1 functions as a key regulatory molecule that is required for normal growth and development of bone and cartilage.
\end{abstract}

Keywords: dentin matrix protein 1, mineralization, osteoblast, chondrocytes, transgenic mice

\section{Introduction}

Bone formation is a complex process that requires bone growth and remodeling. The extracellular matrix of hard tissues is largely comprised of type I collagen and noncollagenous proteins (NCPs). ${ }^{1,2}$ Some of the predominant NCPs of the bone matrix that play a functional role in osteoblast differentiation and matrix mineralization are dentin matrix protein 1 (DMP1), osteopontin, osteonectin, matrix Gla protein, bone sialoprotein, and osteocalcin. Several of these NCPs exert a temporal and spatial influence on oriented hydroxyapatite growth. Among the NCPs, there exists a seminal matrix protein termed DMP1, which was first cloned from a rat cDNA library. ${ }^{3}$ Though the nomenclature implies a function in the dentin matrix, the molecule has also been isolated in osseous tissue and has thus been hypothesized to also have a function in the development and ossification of bone. ${ }^{4,5}$ Following the cloning of rat DMP1, several studies have been undertaken with the goal of characterizing the molecule and determining its function in the biology of calcified tissues. ${ }^{6-8}$ Defining the process of hard tissue formation is central to understanding the process of osteogenesis and subsequently, related pathological entities.

DMP1 is a $58 \mathrm{kDa}$ phosphoprotein with a $\mathrm{pI}$ of 3 found in bone and dentin matrix. ${ }^{3}$ In the bone, DMP1 expression is evident throughout the process of ossification..$^{9,10}$ submit your manuscript $\mid$ www.dovepress.com

Dovepress 
The first evidence for a functional role for DMP1 in mineralization, resulted from in vitro studies, wherein DMP1 was shown to nucleate hydroxyapatite. ${ }^{711}$ However, DMP1-null mice showed no apparent skeletal or tooth phenotype during early development, suggesting functional redundancy due to overlapping expression with other noncollagenous proteins like BAG-75. Feng and colleagues have demonstrated that DMP1-null mice have defective osteocyte maturation, leading to pathological changes in bone mineralization and defects in cartilage formation. ${ }^{12-14}$

In the present study, we examined the biological function of DMP1 in vivo by generating a transgenic (TG) mouse overexpressing DMP1. A cytomegalovirus (CMV) promoter was used as it is reputed to be one of the strongest and contains most promiscuous regulatory elements for directing high transgene expression. ${ }^{15,16}$ In this study, we sought to characterize the skeletal phenotype of the TG mice overexpressing DMP1.

\section{Materials and methods DNA constructs}

The CMV-DMP1 plasmid was generated by first polymerase chain reaction (PCR) amplifying the rat DMP1 cDNA with the coding region and 3' untranslated region. This fragment was then cloned into $\mathrm{KpnI}$ and XhoI sites of the pcDNA 3.1+ vector (Invitrogen, Carlsbad, CA, USA) and digested with Bgl II and Pvu II to release the cassette containing the CMV-DMP1 and bovine growth hormone poly A transgene. The released DNA fragment (transgene) was microinjected into mouse blastocysts.

\section{Generation and genotyping of TG mice}

Microinjections into CD-1 (Charles River Laboratories, Wilmington, MA, USA) outbred mice were performed at the University of Illinois at Chicago Transgenic Facility under institutionally approved protocols. The $\mathrm{F}_{0}$ DMP1-positive lines were identified by isolating genomic DNA from tail fragments using DNeasy tissue kit (Qiagen, Valenica, CA, USA). PCR was carried out with CMV-specific primers. Briefly, $100 \mathrm{ng}$ of genomic DNA was used in the PCR reaction with 35 cycles of $94^{\circ} \mathrm{C}$ for $30 \mathrm{sec}, 56^{\circ} \mathrm{C}$ for $30 \mathrm{sec}$, and $72{ }^{\circ} \mathrm{C}$ for $30 \mathrm{sec}$. Genotyping was carried using the following specific primers for $\mathrm{CMV}$ promoter.

CMV Forward 5'--- GTTCATAGCCCATATATGGAGTTCCGCG --- 3'

CMV Reverse 5' ----- GACCTCCCACCGTACACGCCTAC ----- 3'.
Southern blot analysis was also performed to confirm the presence of DMP1 transgene.

\section{Western blot analysis}

Total protein from liver, muscle, and heart tissue samples were extracted with T-PER lysis buffer (Pierce Biotechnology, Rockford, IL, USA). Briefly, $0.1 \mathrm{~g}$ of fresh tissue from new born animals were homogenized in $2 \mathrm{ml}$ of T-PER lysis buffer and centrifuged at $15,000 \times \mathrm{g}$ for $15 \mathrm{~min}$ and the supernatant collected. Western blot analysis was performed with affinity-purified DMP1 antibody. Total protein from the bone tissue was isolated by homogenizing the femurs from three-day-old animals in the presence of liquid nitrogen and extracted with 0.5 M EDTA (ethylenediaminetetraacetic acid) at $\mathrm{pH} 7.5$ in the presence of a protease inhibitor mixture.

\section{Histological analysis}

The hind limb and the mandibles were dissected out from the TGs $(n=5)$ and the wild type (WT; $n=5)$ at four weeks of age. The tissues were decalcified and embedded in paraffin. Sections were cut at $5 \mu \mathrm{m}$ thickness and treated with various histological stains. Hematoxylin and eosin (H\&E) stain was used for outlining the general tissue architecture, Masson's trichrome was used to detect the presence of connective tissue fibers and Safrannin O/Fast green was used to detect proteoglycans.

\section{Skeleton preparation and analysis}

Skeletons were prepared as described by Tylzanowski and colleagues ${ }^{17}$ Briefly, mice at three days, five days, and two weeks postnatal were sacrificed with carbon dioxide $(n=5)$. The nonskeletal tissues were digested with $2 \%$ potassium hydroxide and then fixed in $95 \%$ ethanol for 72 hours, fixed in acetone for 24 hours and stained with alcian blue for 36 hours. The stained bodies were then washed in $95 \%$ ethanol and placed in a $95 \%$ ethanol bath for 48 hours, changing the solution after 24 hours. Photographs of the caudal vertebral column were taken prior to clearing and counterstaining using a 5 megapixel Fuji digital camera (Fujifilm, Valhalla, NY, USA). The bodies were then placed in a $1 \%$ potassium hydroxide solution until clearing was evident, followed by alizarin red counterstaining for 24 hours. Secondary clearing was performed with $1 \%$ potassium hydroxide for a minimum of 48 hours, after which the specimens were treated with a series of $2 \%$ potassium hydroxide-glycerol solutions every 12 hours in the KOH-glycerol ratios of 80:20, 60:40, 40:60, and 20:80. Final storage was in 100\% glycerol. Images of cleared specimens were recorded with a 5 megapixel Fuji digital camera on a Kaiser light box. Cartilaginous tissues 
were detected by alcian blue staining and ossified tissues by alizarin red.

\section{Radiographic analysis}

Phenotypic changes were also monitored by a noninvasive, high-magnification X-ray analysis to characterize the bones of the craniofacial region of TG and WT four-week-old animals $(n=5)$ via high-resolution digital radiography using a Faxitron model MX-20 digital radiography machine (Faxitron Corporation, Lincolnshire, IL, USA). Specimens subject to comparison were exposed concurrently on the digital sensor and the radiographic densities of the craniofacial region compared between the WT and the TG animals.

\section{Bone formation rate}

Bone formation rate was quantified using flurochrome injections at two different time points in male mice ( $\mathrm{TG}, \mathrm{n}=7$; WT, $n=8)$. At day 14 , the mass of each mouse was determined and $0.09 \mathrm{mg}$ xylenol orange per gram of mouse tissue was administered. At day 24 , the mice were injected with a solution of $0.01 \mathrm{mg}$ tetracycline per gram of mouse tissue. At day 28, the mice were sacrificed and the femurs removed. The femurs were rinsed with phosphate-buffered saline (PBS), fixed overnight in alcohol formalin and dehydrated with successive baths of $70 \%, 80 \%, 96 \%$, and $100 \%$ ethanol at $4{ }^{\circ} \mathrm{C}$. Following dehydration, the bones were mounted in epoxide resin. Ground sections of femurs (cross-sectional) at the proximal diaphysis were prepared to approximately 150 micron thickness. Ground sections were then photographed under a Zeiss Observer D.1 flourescence microscope/AxioCam MRc digital camera (Carl Zeiss, Maple Grove, MN, USA) utilizing GFP (green) and $\mathrm{Cy} 3$ (red) wavelength filter cubes at $5 \mathrm{x}$ magnification. The Axiovision ( $v$ 4.6.3.0) imaging software was used to trace the xylenol orange flurochrome band and exterior cortical plate of the tetracycline-stained portion of each section. The area (in square micrometers) between the two bands was calculated via the integrated software algorithms and the mean bone formation rate along with the standard deviation was calculated to three significant figures for both WT and TG mice.

\section{Immunohistochemical analysis}

Immunohistochemical analysis was performed on serial sections with Col X antibody (kind gift from Dr Horton). The paraffin sections were deparaffinized in xylene, rehydrated, and rinsed three times (5 min each) in PBS ( $\mathrm{pH} 7.4)$ prior to quenching of endogenous peroxidase activity $\left(3 \% \mathrm{H}_{2} \mathrm{O}_{2}\right.$ in PBS at room temperature for $10 \mathrm{~min}$ ). The sections were then rinsed three times ( 5 min each) in PBS and then blocked with $10 \%$ normal goat serum for $45 \mathrm{~min}$ at room temperature. The sections were again rinsed in PBS solution and incubated with primary antibodies against type $\mathrm{X}$ Col at 1:250 dilution (overnight at $4{ }^{\circ} \mathrm{C}$ ). A negative control was included by using an equal concentration of rabbit pre-immune serum as primary antibody. Following incubation with the primary antibody, the tissue sections were rinsed three times (15 min each) in PBS and then incubated with an alkaline phosphatase conjugated goat anti-rabbit immunoglobulin (1:500) for $60 \mathrm{~min}$ at room temperature. Stained sections were digitally archived using a Leica DMLB light microscope (Leica Microsystems, Wetzlar, Germany) and MagnaFire software (Optronics, Goleta, CA, USA).

\section{Stimulation of rat bone marrow stem cells with DMPI}

Rat bone marrow stem cells (RBMSCs) were obtained from Tulane Cancer centre. These cells were cultured in alpha minimal essential medium (MEM) supplemented with l-glutamine, 5\% fetal bovine serum (FBS) and 1\% penicillin-streptomycin. The cells were seeded in 6-well plates and grown to $70 \%$ confluency. Media was changed every two days. The cells were cultured in complete medium supplemented with $0.5 \%$ FBS $12-16$ hours before the start of the experiment. The cells were treated with 0 (control), 250 or $500 \mathrm{ng} / \mathrm{ml}$ of recombinant DMP1 for varying time points (two and five days). Total RNA was isolated after DMP1 treatment using TriZol (GIBCO, Carlsbad, CA, USA). $3 \mu \mathrm{g}$ of DNaseI treated total RNA was reverse transcribed using Superscript III (GIBCO) for $90 \mathrm{~min}$ at $50{ }^{\circ} \mathrm{C}$. PCR reaction was carried out using primers for $\mathrm{Cbfa} 1, \mathrm{BMP} 2$, OCN, and COL1. PCR products were amplified for 35 cycles (30 sec at $94{ }^{\circ} \mathrm{C}, 30 \mathrm{sec}$ at $56^{\circ} \mathrm{C}$, and $30 \mathrm{sec}$ at $72{ }^{\circ} \mathrm{C}$ ) in a DNA thermal cycler (Eppendorf, Westbury, NY, USA). The DNA products were resolved on agarose gels and visualized with ethidium bromide staining. The intensity of the PCR products was quantitated using National Institutes of Health software. The expression level was normalized with GAPDH (glyceraldehyde 3-phosphate dehydrogenase). Gene-specific primers were designed from the DNA sequences at the National Centre for Biotechnology Information gene databank.

\section{Statistical analysis}

Data are expressed as the mean \pm standard deviation of at least three independent experiments or five animals per group, respectively. Statistical analysis was performed 
by one-way analysis of variance, followed by Student's unpaired $t$-test. $P$ values less than 0.05 were considered significant.

\section{Results}

\section{Generation of a TG mouse overexpressing DMPI}

Three TG mouse lines with different integration of the transgene were generated. Two of these lines expressed high levels of the transgene based on the PCR results (data not shown). Because the two TG lines showed no phenotypic differences, line 129 was used for further analysis. PCR results (Figure 1) demonstrated a fivefold increase in the transgene expression
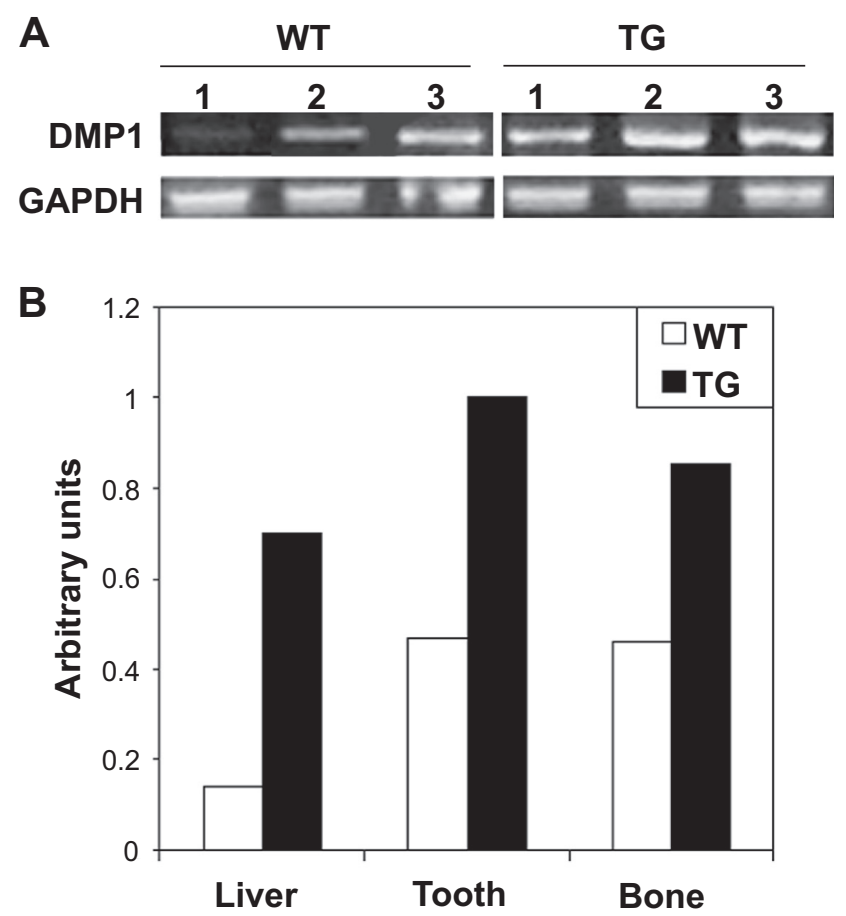

C

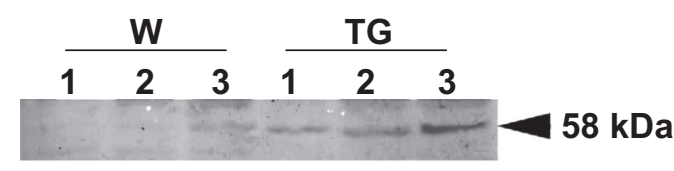

Figure I Characterization of DMPI overexpressing TG mice. A) Total RNA was isolated and subjected to RT-PCR and amplified using DMPI primers from published sequence. B) Quantification of the RT-PCR expression levels in the wild type and TG mice. Note increased expression levels in the teeth and bone of the TG line. C) Proteins were isolated from tissues by quick freezing in liquid nitrogen and were resolved on a 10\% SDS-polyacrylamide gel and transferred to nitrocellulose. The membrane was probed with anti-DMPI antibody. Tissues from TG animals showed increased expression of DMPI.

Notes: Note the absence of DMPI expression in the wild type tissues. Lane I = liver tissue; 2 = heart tissue; 3 = bone.

Abbreviations: DMPI, dentin matrix protein I; RT-PCR, reverse transcriptasepolymerase chain reaction; SDS, ; TG, transgenic. in the liver and a twofold increase in bone and teeth when compared with the WT. Western blotting analysis further confirmed high levels of expression of DMP1 in soft and mineralized tissues (Figure 1C). These results confirmed that in the TG mouse line, DMP1 expression is under the control of the CMV promoter.

\section{TG mice exhibit abnormalities in the skeletal phenotype}

Body weight comparison between the TG and WT mice demonstrate higher body mass with age in the TG mice, indicating phenotypic changes during growth and development (data not shown). Consequences of DMP1 overexpression on skeletal abnormalities were determined by staining whole skeletal preparations with alcian blue and alizarin red. Skeletal staining of the three-day-old mice revealed accelerated mineralization of the intramembranous bones. In the craniofacial area, enhanced mineralization around the suture was present in the TG line when compared with the WT line (Figure 2A). In the TG, the peripheral borders of the flat bones of the cranium displayed a sharp, irregular appearance, which suggests more aggressive mineralization when compared with the WT (Figure 2A).

High-resolution digital radiography of four-week-old TG mice revealed increased radiodensity in the craniofacial region in comparison to the WT mice (Figure 2B). Specifically, increased radiodensity was noted in the occipital area of the skull and the palatal area. In addition to this, instances of increased radiodensity were noted in the bony labyrinth of the temporal bone as well as the nasal conchae (Figure 2B). This was verified by performing concurrent imaging of the specimens on the digital sensor. This data suggests that DMP1 regulates the osteoblastic activity responsible for intramembranous bone formation.

Skeletal staining of the five-day-old mice revealed differences between the TG and WT in the ossification of the carpal bones (Figure 3A, arrows). By day 5, the WT demonstrated initial mineralization of only the trapezoid, pisiform and/or triquetral bones. In contrast, the TG line displayed ossification of these bones as well as the capitate, hamate, falciform carpal, and scaphoid. In addition, ossification centers in the distal epiphyseal plate of the 5 th metacarpal have appeared in the TG line by day 5, but have not developed yet in the WT. Thus, increased ossification of the carpal bones were observed. Calcification of the caudal portion of the axial skeleton also showed increased ossification in the TG line (Figures 3B and C). TG mice had an average of four additional tail vertebrae that had begun 
A

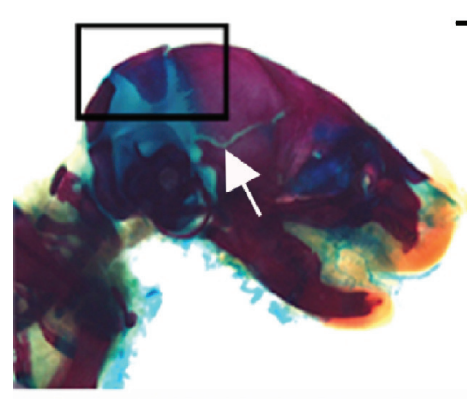

WT 3 Days

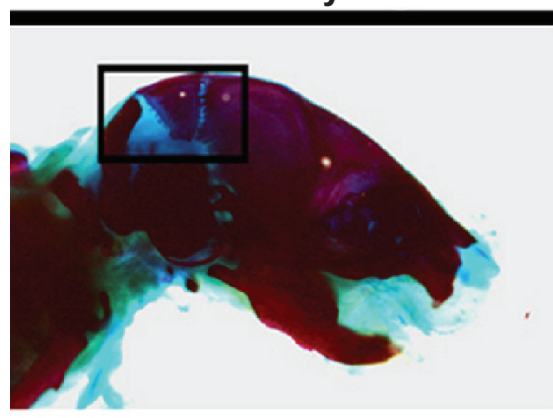

TG 3 Days

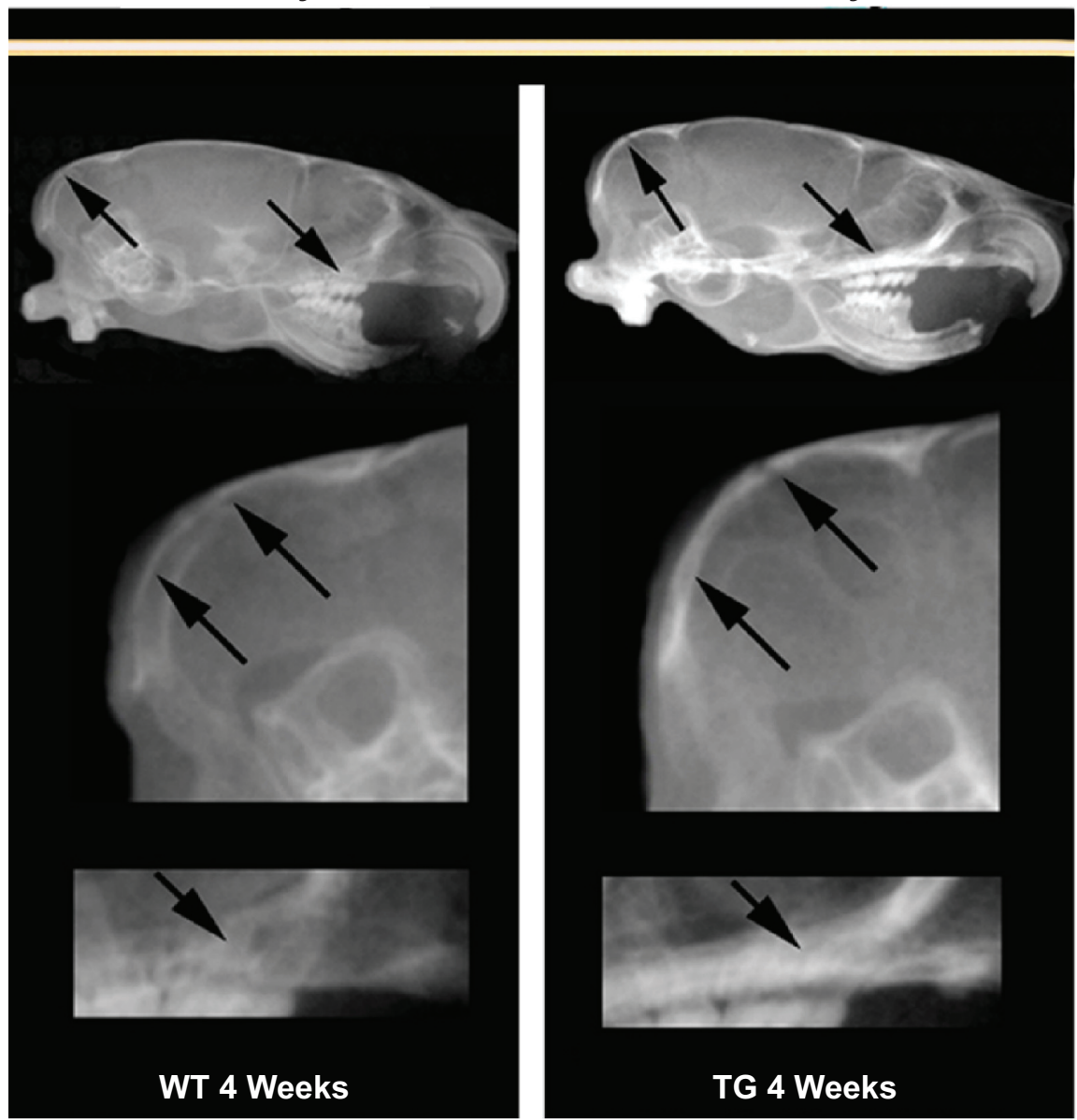

Figure 2 DMPI transgenic (TG) mice exhibit accelerated mineralization of the intramembranous cranial bones. A) Skeletal staining with alizarin red and alcian blue display aggressive mineralization (arrows) of skull sutures in three-day-old TG when compared with the wild type (WT). Bar $=5 \mathrm{~mm}$. Boxed areas are magnified and shown on the right. Bar $=2 \mathrm{~mm}$. B) High resolution digital radiography of four-week-oldTG and WT mouse skulls focusing on the occipital and palatal region of the craniofacial area.Arrows indicate areas of increased radiodensity in the occipital (middle) and palatal areas (bottom).

Abbreviation: DMPI, dentin matrix protein I. 


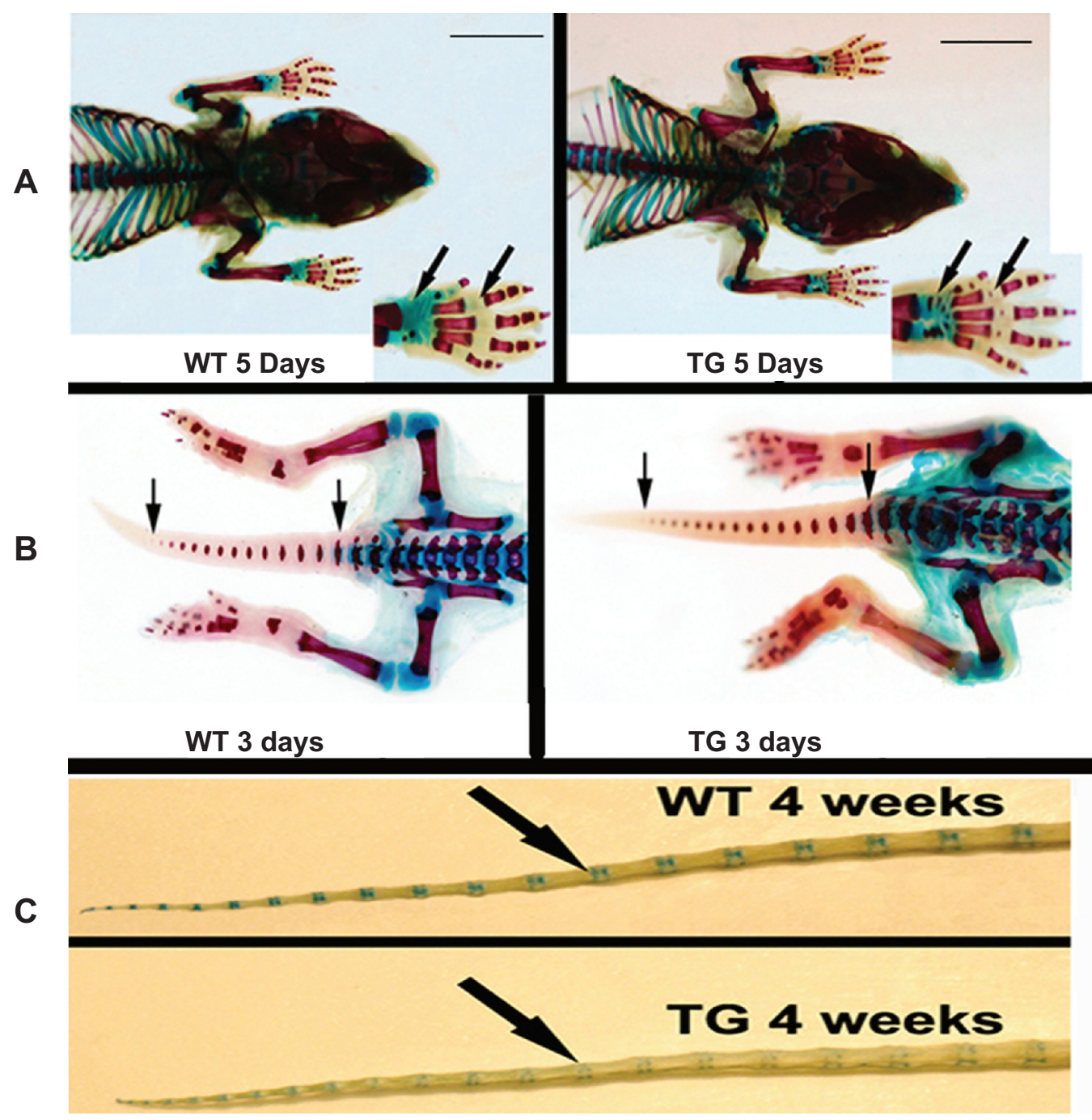

Figure 3 Magnified regions of the whole mount skeletons of wild type (WT) and transgenic (TG) mice stained with alcian blue (for cartilage) and alizarin red (for calcified tissues). A) Composite image of five-day-old TG mice versus WT mice displaying accelerated mineralization of the carpal and metacarpal bones (arrows). Bar $=10$ mm B) Composite image show accelerated mineralization of the caudal vertebrae (arrows) in TG versus WT mice. Note increase in the number of the tail vertebrae when compared to the control. C) Alcian blue staining of the caudal region of four-week-old specimens. Arrows denote decreased alcian blue staining indicating less proteoglycans in the intervertebral discs of TG versus WT mice.

mineralizing earlier versus the WT at the same time point (17.5 versus 13.6). Skeletal staining of the four-week-old mice revealed decreased proteoglycan content in the intervertebral discs of TG mice, as demonstrated with alcian blue staining (Figure 3B). Upon clearing with potassium hydroxide, the proteoglycan deficiency in the TG mice remained evident, both in the two- and the four-week-old mice (Figure 3C). Overall, increased alizarin red staining in the skeleton of the TG mice demonstrates enhanced mineralization.

\section{TG mice exhibit abnormalities}

\section{in the growth plate}

Histological H\&E staining of the skeleton revealed a narrower cartilaginous growth plate in the epiphysis of the
TG mice at four weeks of age (Figure 4A). In addition to this, differences were noted in the cellular arrangement and morphology of the cartilaginous growth plate associated with the head of the femur (Figure 4A). The TG mouse displayed altered cell morphology and architecture, specifically lacking the classical columnar arrangement noted in the zone of proliferation and maturation stage and instead displayed a prevalence of hypertrophic, calcifying cartilage cells. Presence of hypertrophic chondrocytes was confirmed by the presence of type X collagen antibody (Figure 4C). Moreover, the bone around the articular surface was highly mineralized (Figure 4B). Saffrannin O staining clearly demonstrated that the bone volume in the primary spongiosa just beneath the growth plate of the tibiae was significantly 
increased resulting from the early differentiation of the chondrocytes (Figure 4B). These data suggest that the early hypertrophic phenotype of the chondrocytes is due to the overexpression of DMP1 in these cells.

\section{Assessment of bone formation rate}

Analysis of the mean area of the bone formed (in square micrometers) between serial xylenol orange and tetracycline injections (Figures 5A and B) in the femurs revealed that the phenotype seen in the TG mice is caused by an increase in bone formation rate. Over the 14-day study interval, the TG mice displayed an average of 521,000 $\mu \mathrm{m}^{2}$ (standard error $[\mathrm{SE}] \pm 58,100 \mu \mathrm{m}^{2}$ ) of increased bone area, while the WT animals exhibited an average of $468,000 \mu \mathrm{m}^{2}$ (SE $\pm 84,600 \mu \mathrm{m}^{2}$ ) of increased bone area (Figure $5 \mathrm{C}$ ). Thus the TG animals exhibited an average of $11.3 \%$ increase in the bone formation rate over the WT animals up to four weeks of age.

\section{Terminal differentiation of RBMSCs to osteoblasts in the presence of DMPI}

To define the molecular mechanism for the accelerated osteoblast differentiation that was observed in the TGs, rat bone marrow stem cells were treated with recombinant DMP1 for specified time periods. Reverse-transcriptase-PCR (RT-PCR) analysis (Figure 6) showed an upregulation of lineage specific early markers such as Cbfa1, signaling molecules like BMP2, and late markers for mature osteoblasts such as type I collagen and osteocalcin. As shown in Figure 6A, BMP2, Cbfa1, type I collagen, and OCN expression levels increased within two days and osteocalcin and BMP2 collagen expression increased several folds within five days (Figure 6B). This osteoblast lineage specific expression was further confirmed by accelerated mineralized nodule formation in RBMSCs treated with DMP1 (Figures 6C and D). Taken together, these results indicate that DMP1 influences the differentiation of bone marrow stem cells into functional osteoblasts.

\section{Discussion}

DMP1 is a biologically-active molecule and has been implicated to have a regulatory function during early osteoblast and odontoblast differentiation as well as during the formation of the calcified extracellular matrix, by various means, including the development of a DMP1-null mouse. ${ }^{14,18-23}$ Inactivation of the DMP1 gene did not induce gross morphological abnormalities in bone and dentin during early development, partly because DMP1 has

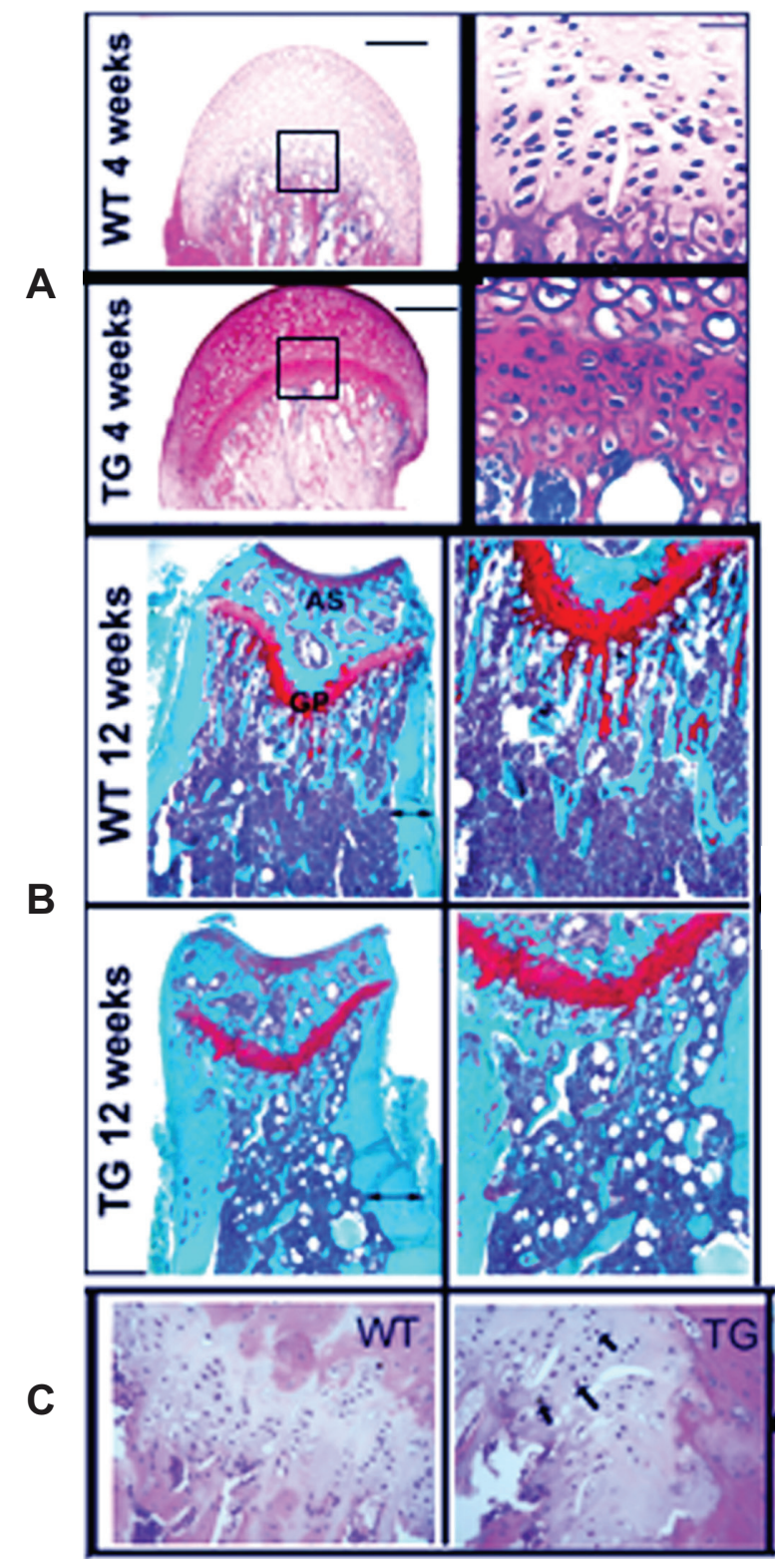

Figure 4 Impaired growth plate chondrocyte proliferation and maturation. A) Representative micrographs of hematoxylin and eosin (H\&E) stained sections of proximal tibias of four-week-old mouse femurs. The transgenic line (TG) displayed altered columnar arrangement and altered morphology of the hypertrophic zone and smaller width of the growth plate. The wild type (WT) displayed normal columnar architecture characteristic of the normal zones of the growth plate (GP). Bar $=500 \mu \mathrm{m}$. Magnified images of the boxed areas are shown on the right. Bar $=50 \mu \mathrm{m}$. B) Representative micrograph of Safrannin-O stained section of the proximal growth plate of four-week-old mouse tibias. Bone adjacent to the articular surface of the TG line is more solid and contiguous from the articular surface (AS) to the growth plate (GP). In the WT, the bone appears less contiguous, and larger marrow cavity is observed. Note the thickness of the cortical bone in TG mice when compared with the WT mice as indicated by the arrows in the cortical bone area. C) Representative micrographs of the tibial section of WT and TG mice stained immunohistochemically for type $X$ collagen. Note increased expression of $\mathrm{Col} X$ in the hypertrophic chondrocytes of the TG mice (Magnification, $x 400$ ).

several homologies to other proteins. ${ }^{24}$ The first 10 amino acid residues are identical to the $\mathrm{N}$-terminal sequence of bone glycoprotein BAG-75 (bone acidic glycoprotein -75) and to the first eight residues of the core protein of a bone 

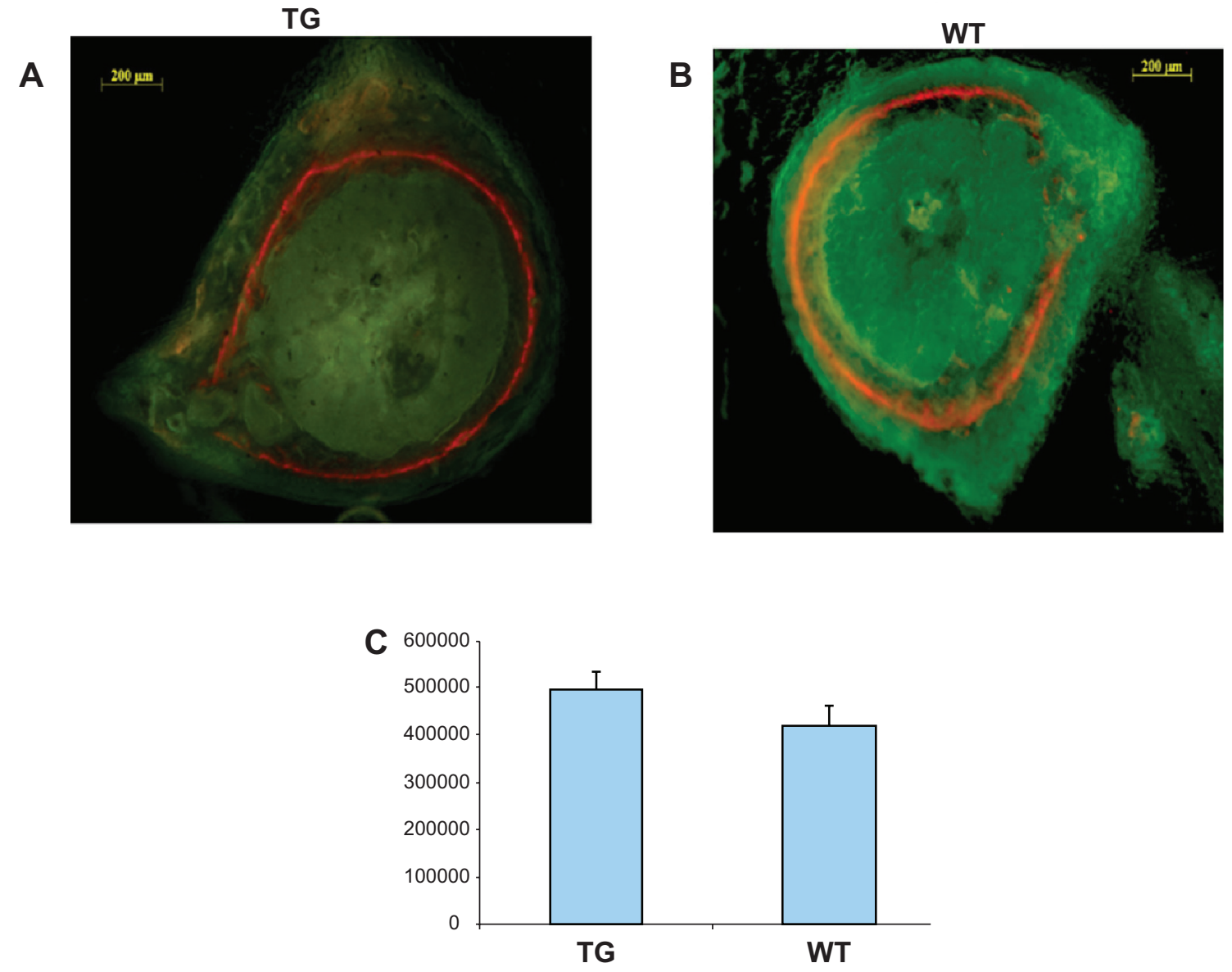

Figure 5 Bone formation rate. Bone formation rate was obtained using fluorescent calcium binding dyes xylenol orange and tetracycline which were used sequentially to label new bone deposition in wild type (WT) (a) and transgenic (TG) (b). Graphical representation of bone formation rate is shown in (c).

proteoglycan designated as HS-PGIII. ${ }^{25}$ Moreover, DMP1 belongs to a class of small, integrin binding proteins which is currently represented by the glycophosphoproteins encoded by 5 genes clustered within a $372 \mathrm{~kb}$ region of human chromosome 4 called the SIBLING (small integrin-binding ligand, N-linked glycoprotein) family. ${ }^{26}$ Therefore, the phenotype in DMP1 knockout mice might be due to unexpected compensatory or functional redundancy mechanisms that might be activated when a gene is ablated. In this study, a gain-of-function approach was employed to define the in vivo function of DMP1, by generating a DMP1-overexpressing TG mouse using a CMV promoter. As anticipated, the TG mice exhibited accelerated mineralization in osseous tissues.

On a microscopic level, examination of the mouse femurs revealed alterations in the morphology of the bony tissues. Specifically, the growth plate of the DMP1-overexpressing mice was found to be narrower and displayed altered chondrocyte morphology and architecture. In addition, the growth plate lacked the classical well-defined columnar arrangement of the chondrocytes that progress through proliferative, prehypertrophic and terminally differentiating hypertrophic stages. Instead, the epiphyseal growth plate displayed an accumulation of disorganized hypertrophic chondrocytes. This indicates that DMP1 overexpression was responsible for accelerated chondrocyte hypertrophy that resulted in depositing a cartilaginous matrix. The matrix provided the template for differentiating osteoblasts from the bone marrow to form trabecular bone at the site of the bone collar. We have demonstrated earlier that DMP1 upregulated the expression of Cbfa1 in mouse calvarial MC3T3-E1 cells. ${ }^{27}$ Thus enhanced expression of Cbfal in the growth plate chondrocytes might accelerate chondrocyte hypertrophy. This is in agreement with the results that overexpression of Cbfa 1 accelerated chondrocyte maturation and endochondral ossification while Cbfa1 deficient mice show limited cartilage calcification. ${ }^{28-31}$ On the other hand, DMP1 null mice exhibited a severe defect during postnatal chondrogenesis and presented a chondroplasia-like phenotype. ${ }^{13}$ Taken together, these data indicate that DMP1 can enhance chondrocyte 

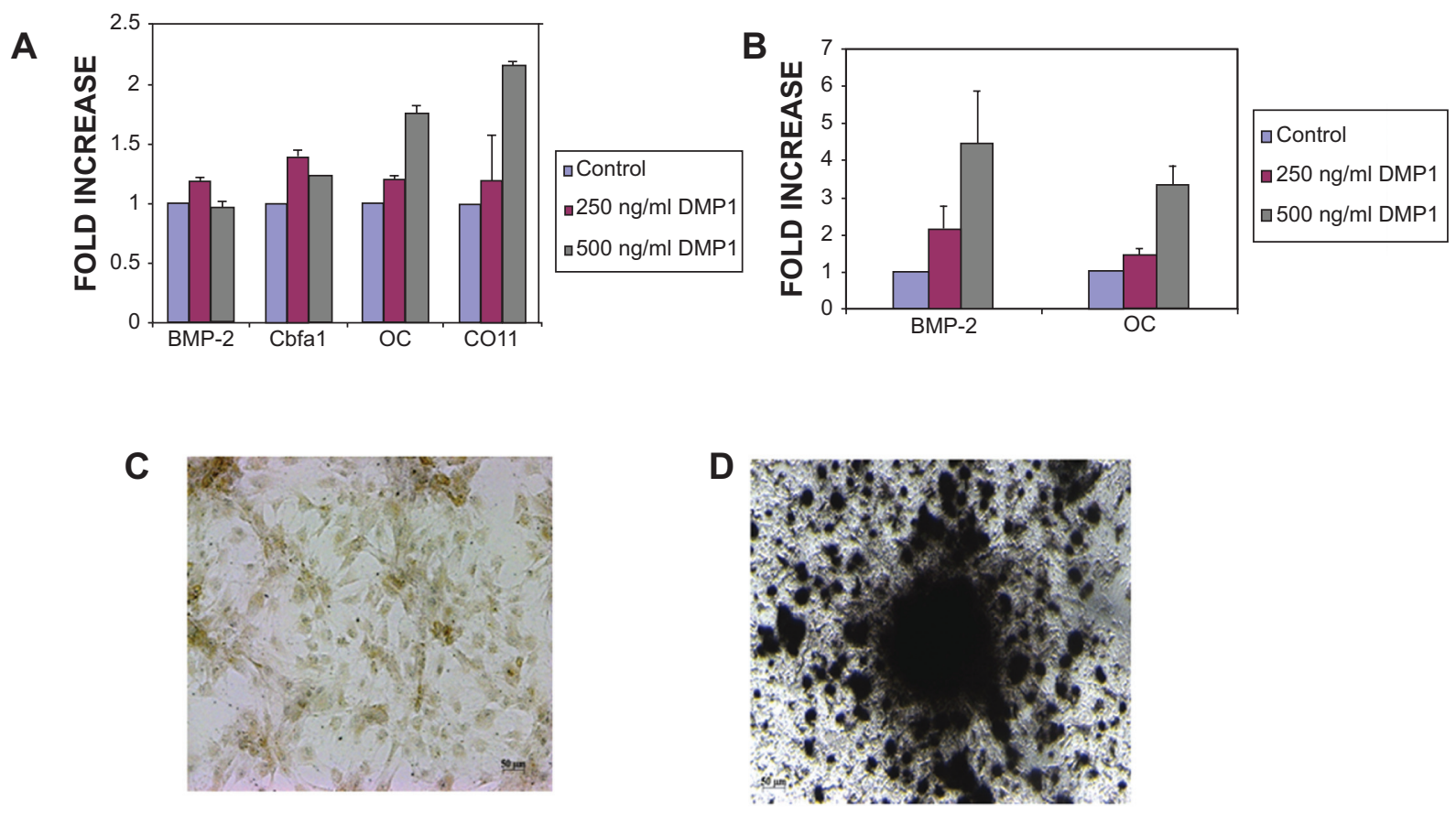

Figure 6 Role of DMPI in osteoblastogenesis. Rat bone marrow stem cells (RBMSCs) were treated with either 250 or $500 \mathrm{ng}$ of DMPI for two A) or five days B). Total RNA was extracted and the expression of Cbfa I, BMP2, OCN, and Coll (primer sequences were obtained from GenBank) were detected by semi-quantitative reverse-transcriptasepolymerase chain reaction. Note expression levels of "early" and "late" markers increased with the commitment and differentiation of RBMSCs to the osteogenic lineage. Mineralized nodule assay was performed by treating RBMSCs with $500 \mathrm{ng}$ DMPI for 30 days. von Kossa staining shows the presence of mineralized nodules only in RBMSCs treated with DMPI D) and not in untreated cells $\mathbf{C}$ ).

Abbreviation: DMPI, dentin matrix protein I.

hypertrophy and this may be associated with upregulation of Cbfa1.

Skeletal staining revealed a similar pattern of decreased cartilaginous tissue formation at the macroscopic level in the DMP1 TG mice. Reduced alcian blue staining of the intervertebral discs of the TG mice clearly demonstrates that the cartilage forming mechanisms were altered, facilitating rapid mineralization. This was further confirmed after counterstaining with alizarin red. In three-day-old TG lines, evidence for enhanced mineralization was further provided by the aggressive suture closure in the TG line and mineralization of the caudal vertebrae. By day 5 , developing TG mice displayed aggressive mineralization of the carpal and metacarpal bones, which provide evidence that overall mineralization, increased rapidly at the active ossification centers during early skeletal development. This increased osteoblast differentiation was reflected in the extensive mineralization at the bone collar in the TGs. Consistent with the observed histological and staining data, high resolution digital radiography revealed increased radiodensity of the craniofacial bones in the TG mice. Dynamic histomorphometric analysis revealed that the cortical bone area formed over a 14-day period in the TG mice was greater than the WT mice. On average, TG mice displayed a $11.1 \%$ increase in bone formation over the control animals. In contrast, the DMP1 knockout mice exhibited decreased endochondral ossification and the mice had defective osteocyte maturation leading to rickets and osteomalacia. ${ }^{14,15}$ Thus, the presence of DMP1 in TG mice consistently developed a propensity for accelerated bone formation during early development.

Our analysis with the bone marrow stem cells further demonstrate that the mineralized phenotype could be due to the commitment and accelerated differentiation of these cells to the osteoblast lineage. Thus, the enhanced mineralization phenotype of the femurs at four weeks is consistent with DMP1 as a positive regulator of mineralized matrix formation. This finding corroborates well with our earlier published data that overexpression of DMP1 accelerates maturation of preosteoblasts to fully differentiated osteoblasts accompanied by upregulation of target genes that accelerate osteoblast differentiation. Similar results were obtained when ectopic expression of the transcription factor Fra-1 in TG mice led to increased bone mass by regulating bone matrix production by osteoblasts and chondrocytes. ${ }^{32}$

In conclusion, our data suggests that robust mineralization observed in the DMP1 overexpressing TG mice could be correlated with the active synthesis of transcription factors, 
signaling molecules and matrix components required for the assembly of the mineralized matrix.

\section{Acknowledgments}

We thank Verna J Brown for histological staining. We also thank Dr William Horton for kindly providing us with the collagen $\mathrm{X}$ antibody. This work was supported by NIH grant DE 11657. The authors report no conflicts of interest in this work.

\section{References}

1. Boskey AL. Matrix proteins and mineralization: an overview. Connect Tissue Res. 1996;35(1-4):357-363.

2. Veis A. Mineral-matrix interactions in bone and dentin. J Bone Miner Res. 1993;8(Suppl 2):S493-S497.

3. George A, Sabsay B, Simonian PA, Veis A. Characterization of a novel dentin matrix acidic phosphoprotein. Implications for induction of biomineralization. J Biol Chem. 1993;268(17):12624-12630.

4. Kalajzic I, Braut A, Guo D, et al. Dentin matrix protein 1 expression during osteoblastic differentiation, generation of an osteocyte GFP-transgene. Bone. 2004;35(1):74-82.

5. D'Souza RN, Cavender A, Sunavala G, et al. Gene expression patterns of murine dentin matrix protein 1 (Dmp1) and dentin sialophosphoprotein (DSPP) suggest distinct developmental functions in vivo. J Bone Miner Res. 1997;12(12):2040-2049.

6. Toyosawa S, O'hUigin C, Klein J. The dentin matrix protein 1 gene of prototherian and metatherian mammals. J Mol Evol. 1999;48(2):1 60-167.

7. He G, Dahl T, Veis A, George A. Nucleation of apatite crystals in vitro by self-assembled dentin matrix protein 1. Nat Mater. 2003;2(8):552-558.

8. Maciejewska I, Qin D, Huang B, et al. Distinct compartmentalization of dentin matrix protein 1 fragments in mineralized tissues and cells. Cells Tissues Organs. 2009;189(1-4):186-191.

9. Hao J, Zou B, Narayanan K, George A. Differential expression patterns of the dentin matrix proteins during mineralized tissue formation. Bone. 2004;34(6):921-932.

10. Jüppner H. Novel regulators of phosphate homeostasis and bone metabolism. Ther Apher Dial. 2007;11(Suppl 1):S3-S22.

11. Tartaix PH, Doulaverakis M, George A, et al. In vitro effects of dentin matrix protein-1 on hydroxyapatite formation provide insights into in vivo functions. J Biol Chem. 2004;279(18):18115-11820.

12. Feng JQ, Ward LM, Liu S, et al. Loss of DMP1 causes rickets and osteomalacia and identifies a role for osteocytes in mineral metabolism. Nat Genet. 2006;38(11):1310-1315.

13. Ye L, Mishina Y, Chen D, et al. Dmp1-deficient mice display severe defects in cartilage formation responsible for a chondrodysplasia-like phenotype. J Biol Chem. 2005;280(7):6197-6203.

14. Feng JQ, Scott G, Guo D, et al. Generation of a conditional null allele for Dmp1 in mouse. Genesis. 2008;46(2):87-91.

15. Kachi S, Esumi N, Zack DJ, Campochiaro PA. Sustained expression after nonviral ocular gene transfer using mammalian promoters. Gene Ther. 2006;13(9):798-804.
16. Liu YH, Tang Z, Kundu RK, et al. Msx2 gene dosage influences the number of proliferative osteogenic cells in growth centers of the developing murine skull: a possible mechanism for MSX2-mediated craniosynostosis in humans. Dev Biol. 1999;205(2):260-274.

17. Tylzanowski P, Mebis L, Luyten FP. The Noggin null mouse phenotype is strain dependent and haploinsufficiency leads to skeletal defects. Dev Dyn. 2006;235(6):1599-1607.

18. Maciejewska I, Cowan C, Svoboda K, Butler WT, D’Souza R, Qin C. The NH2-terminal and $\mathrm{COOH}$-terminal fragments of dentin matrix protein 1 (DMP1) localize differently in the compartments of dentin and growth plate of bone. J Histochem Cytochem. 2009;57(2):155-166.

19. Ravindran S, Narayanan K, Eapen AS, et al. Endoplasmic reticulum chaperone protein GRP-78 mediates endocytosis of dentin matrix protein 1. J Biol Chem. 2008;283(44):29658-29670.

20. Lu Y, Qin C, Xie Y, Bonewald LF, Feng JQ. Studies of the DMP1 57-kDa functional domain both in vivo and in vitro. Cells Tissues Organs. 2009; 189(1-4):175-185.

21. He G, Gajjeraman S, Schultz D, et al. Spatially and temporally controlled biomineralization is facilitated by interaction between self-assembled dentin matrix protein 1 and calcium phosphate nuclei in solution. Biochemistry. 2005;44(49):16140-16148.

22. Ling Y, Rios HF, Myers ER, Lu Y, Feng JQ, Boskey AL. DMP1 depletion decreases bone mineralization in vivo: an FTIR imaging analysis. J Bone Miner Res. 2005;20(12):2169-2177.

23. Narayanan K, Srinivas R, Peterson MC, et al. Transcriptional regulation of dentin matrix protein 1 by JunB and p300 during osteoblast differentiation. J Biol Chem. 2004;279(43):44294-44302.

24. Ye L, MacDougall M, Zhang S, et al. Deletion of dentin matrix protein-1 leads to a partial failure of maturation of predentin into dentin, hypomineralization, and expanded cavities of pulp and root canal during postnatal tooth development. J Biol Chem. 2004;279(18):19141-19148.

25. Srinivasan R, Chen B, Gorski JP, George A. Recombinant expression and characterization of dentin matrix protein 1. Connect Tissue Res. 1999;40(4):251-258.

26. Fisher LW, Fedarko NS. Six genes expressed in bones and teeth encode the current members of the SIBLING family of proteins. Connect Tissue Res. 2003;44(Suppl 1):33-40.

27. Narayanan K, Ramachandran A, Hao J, et al. Dual functional roles of dentin matrix protein 1. Implications in biomineralization and gene transcription by activation of intracellular $\mathrm{Ca}_{2}^{+}$store. $J$ Biol Chem. 2003;278(19):17500-17508.

28. Komori T. A fundamental transcription factor for bone and cartilage. Biochem Biophys Res Commun. 2000;276(3):813-816.

29. Kobayashi H, Gao Y, Ueta C, Yamaguchi A, Komori T. Multilineage differentiation of Cbfa1-deficient calvarial cells in vitro. Biochem Biophys Res Commun. 2000;273(2):630-636.

30. Hoshi K, Komori T, Ozawa H. Morphological characterization of skeletal cells in Cbfa1-deficient mice. Bone. 1999;25(6):639-651.

31. Kim IS, Otto F, Zabel B, Mundlos S. Regulation of chondrocyte differentiation by Cbfa1. Mech Dev. 1999;80(2):159-170.

32. Eferl R, Hoebertz A, Schilling AF, et al. The Fos-related antigen Fra-1 is an activator of bone matrix formation. EMBO J. 2004;23(14):2789-2799.
Orthopedic Research and Reviews

\section{Publish your work in this journal}

Orthopedic Research and Reviews is an international, peer-reviewed, open access journal that focusing on the patho-physiology of the musculoskeletal system, trauma, surgery and other corrective interventions to restore mobility and function. Advances in new technologies, materials, techniques and pharmacological agents are particularly welcome. The journal welcomes

\section{Dovepress}

original research, clinical studies, reviews \& evaluations, expert opinion and commentary, case reports and extended reports. The manuscript management system is completely online and includes a very quick and fair peer-review system, which is all easy to use. Visit http://www.dovepress. com/testimonials.php to read real quotes from published authors. 